\title{
Strategies for enhancing information, motivation, and skills for self-management behavior changes: a qualitative study of diabetes care for older adults in Korea
}

\author{
This article was published in the following Dove Press journal: \\ Patient Preference and Adherence \\ 14 February 2014 \\ Number of times this article has been viewed
}

\section{Suyoung Choi' \\ Misoon Song ${ }^{2}$ \\ Sun Ju Chang ${ }^{3}$ \\ Se-an Kim ${ }^{4}$ \\ 'College of Nursing, Jeju National University, Jeju, Korea; ${ }^{2}$ College of Nursing, and The Research Institute of Nursing Science, Seoul National University, Seoul, Korea; ${ }^{3}$ Department of Nursing Science, Chungbuk National University, Cheongju, Korea; ${ }^{4}$ College of Nursing, Seoul National University, Seoul, Korea}

Purpose: To describe strategies for enhancing information, motivation, and skills related to changes in diabetes self-management behavior among community-dwelling older adults in Korea.

Patients and methods: A total of five focus group interviews (three separate focus groups) were conducted with 12 older adults with type 2 diabetes and five diabetes educators. Qualitative content analysis was used.

Results: "One's own willingness and ability" emerged as a fundamental belief about the strategies for diabetes self-management. Six major themes under three categories were identified. Under the information category, the recurrent themes were: 1) repeatedly offering detailed knowledge regarding self-management, 2) providing information about current health status, and 3) identifying experiential knowledge of blood glucose control. The recurrent themes in the motivation category were: 1) ensuring a positive attitude regarding self-management, and 2) encouragement or feedback from significant others. Furthermore, in the skills category, we found that the following theme emerged: hands-on skills training with numerical standards.

Conclusion: This study identified six tailored strategies for enhancing information, motivation, and skills for diabetes self-management behavior changes. These strategies can be used in the development of a culturally sensitive diabetes self-management program for older adults.

Keywords: type 2 diabetes mellitus, self-care, aged, focus group, geriatric nursing

\section{Introduction}

The increasing prevalence of diabetes among older adults is a global trend. ${ }^{1,2}$ The prevalence of diabetes in those aged over 65 years old in Korea has increased from $16.6 \%$ to $22.7 \%$ during the past 10 years,${ }^{3}$ and the proportion of older adults living with diabetes will continue to increase with the accelerated aging of the Korean population. In the long-term, diabetes causes many complications, leading to dysfunction of the heart, kidneys, eyes, blood vessels, and nerves, ${ }^{4}$ resulting in increasing functional disability and premature mortality in diabetic older adults. ${ }^{1,5}$

The risk of diabetes-related complications can be reduced when individuals maintain an optimal blood glucose level. ${ }^{6}$ Furthermore, diabetes self-management is essential for achieving and maintaining optimal glycemic control. ${ }^{7,8}$ Self-management refers to actions that people with chronic illnesses can undertake to manage their symptoms, treatment, and lifestyle changes as per the recommendations of health professionals. ${ }^{9}$ According to the American national standards for diabetes self-management education
Correspondence: Misoon Song College of Nursing, Seoul National University, 28 Yeongun-Dong, Chongno-Gu, Seoul I I0-799, Korea Tel +8227408826

Fax +8227648457

Email msong@snu.ac.kr 
(DSME), ${ }^{10}$ the key factors of the diabetes self-management program were to promote the patients' knowledge, motivation, and skills necessary for diabetes self-management. The same notion was supported by the information-motivationbehavioral skills (IMB) model developed for preventing human immunodeficiency virus (HIV) risk behaviors. ${ }^{11}$ The IMB model suggests three core determinants containing informational, motivational, and behavioral skills factors for changing and maintaining health behaviors, and posits a set of causal relationships among these constructs. Thus, if individuals are well informed, well motivated to perform health behaviors such as self-management, and acquire the behavioral skills to act effectively, they will be more adept at achieving positive health outcomes. ${ }^{11-13}$ However, effective strategies to enhance these core determinants for diabetes self-management are unclear. ${ }^{14,15}$

Older adults with diabetes experience difficulties managing diabetes with their coexistent chronic illness due to the complex nature of diabetes self-management, as well as their physical and functional changes because of the aging process. ${ }^{1,16}$ Although a few previous studies have described facilitators of or barriers to diabetes self-management involving a population of diverse ages, ${ }^{5,16-18}$ there is limited knowledge about how older people acquire the information, motivation, and skills to change their diabetes selfmanagement behaviors. Therefore, the effective strategies suggested by older adults to facilitate behavior changes in diabetes self-management will be particularly important. In addition, effective strategies identified by diabetes educators would be valuable, as they have valuable information that will contribute to developing an up-to-date, patient centered, and culturally relevant program that is responsive to participants' needs. ${ }^{10}$ Therefore, it is necessary to investigate the strategies that are successful in enhancing the information, motivation, and skills related to changes in diabetes self-management behavior, not only from older adults with diabetes but also diabetes educators. The integrated knowledge from this study will promote overall success in diabetes self-management.

Thus, this study's purpose was to identify strategies for enhancing the information, motivation, and skills for diabetes self-management behavior changes for community-dwelling older adults in Korea by integrating data from older adults with type 2 diabetes and diabetes educators via focus group interviews. This understanding will assist in identifying effective methods for tailored intervention that focus on the determinants of diabetes self-management behavior changes.

\section{Methods}

\section{Setting and participants}

A purposive sampling strategy was used for recruiting older adults with diabetes and diabetes educators. To recruit potential focus group participants, we contacted nurses at two senior centers and a diabetes educator at a university hospital in Seoul. They approached potential participants to introduce this study to them and asked whether the authors were allowed to contact them. A total of 24 older adults and five diabetes educators were initially selected using the nurses' and diabetes educator's referrals. Additionally, all of the initially selected older adults were participating in diabetes self-management programs or self-help groups within the community-based senior centers. The authors contacted via telephone only those individuals who consented, informing them of the study, and asked them whether they would participate. All potential participants agreed to participate in this study.

To select the older participants who had achieved a higher level of diabetes self-management, the diabetes self-management score of the potential older participants was measured using the Summary of Diabetes Self-Care Activities Questionnaire (SDSCA). ${ }^{19}$ This score's median value among the potential older participants was 40 . The inclusion criteria for older adults were: 1) age 65 or older; 2) diagnosed with type 2 diabetes more than 6 months prior to the study; 3 ) oral hypoglycemic agent or insulin, and lifestyle modification prescribed by a physician; and 4) screened diabetes self-management score over 40 . The inclusion criteria for diabetes educators were nurses and dieticians who had been working over 3 years in DSME.

Consequently, in this study, a total of 12 communitydwelling older adults with type 2 diabetes and five diabetes educators (three nurses and two dieticians) were recruited from two senior centers and a university hospital, respectively.

\section{Data collection}

Focus group interviews were used for data collection. There were three focus groups: seven older adults were recruited from a diabetes self-help group at one senior center (group P1), five older adults were recruited from another senior center (group P2), and five diabetes educators were recruited (group E1).

A total of five focus group interviews (three separate focus groups) were conducted until saturation was reached, ie, when the data occurred so frequently that the authors could anticipate it and no new themes emerged. All five diabetes educators, including three nurses and two dietitians, 
participated in the first and second focus group interviews. The third focus group interview was attended by seven older adults from one senior center's self-help group, and six of them participated in the fourth focus group interview. One older participant could not participate in the fourth focus group interview because of illness. The fifth focus group interview was attended by five older adults from the diabetes self-management program at the other senior center. As a result, the focus group interviews were conducted twice for group P1, once for P2, and twice for group E1. Using multiple focus groups adequately assesses the point of data saturation, with each group interview occurring once or multiple times. ${ }^{20}$ Each focus group lasted 60-80 minutes.

The interview guide was designed to elicit strategies regarding the enhancing of information, motivation, and skills for diabetes self-management behavior changes. The five major interview question areas are presented in Table 1. The focus groups for P1 and P2 discussed strategies related to information, motivation, and skills necessary for diabetes self-management among older people. Similarly, the focus groups for E1 discussed their perceptions related to strategies for enhancing information, motivation, and skills for diabetes self-management; they also discussed their perceptions of facilitators and barriers for the same. All focus group interviews were audio recorded and transcribed verbatim.

\section{Data analysis}

The data were analyzed using the eight-step process of qualitative content analysis. ${ }^{21}$ The unit of analysis was the

\section{Table I Focus group interview guide}

Older adults with type 2 diabetes

I. What is the most important or necessary information to control your blood glucose level?

2. What do you think about motivating factors to start or maintain diabetes self-management in your daily life?

3. What are the most important or necessary behavioral skills to control your blood glucose level?

4. What kinds of things make diabetes self-management easy for you or help you?

5. What kinds of things make diabetes self-management hard or difficult for you?

\section{Diabetes educators}

I. What is the most important or necessary information to improve diabetes self-management in older adults with diabetes?

2. What are the factors that motivate older adults with diabetes to start or maintain diabetes self-management?

3. What are the most important or necessary behavioral skills to control blood glucose level?

4. What makes it easy for older adults to manage their diabetes?

5. What makes it hard or difficult for older adults to manage their diabetes? interview data of each focus group. Each author independently read all transcripts from the focus group interviews several times to obtain the overall sense and reflective notes. The authors developed the three category schemes (information, motivation, and skills). Each author coded all interview data, and coding discrepancies were discussed and resolved during a series of weekly meetings. The codes were incorporated into the categories. The authors described and analyzed the content of the categories within and across the focus groups to develop themes.

\section{Ethical considerations}

Approval to conduct this study was obtained from the Institutional Review Board of College of Nursing in Seoul prior to collecting data for the study. The investigators obtained informed written consent after providing explanation of the study purpose, procedure, benefits, and risks and the right to withdraw at any time prior to or during the interview.

\section{Results}

The twelve older adults participating in this study included seven females and five males, with an age range of 73-85 years $(78.5 \pm 4.2)$ and the SDSCA score range of 43-93 (62.3 \pm 14.2$)$. Most (83.4\%) were being treated with oral hypoglycemic agents only, and the time since diagnosis ranged from 3 to 35 years (15.6 \pm 10.0$)$. The length of experience of the five diabetes educators who participated in this study ranged from 3 to 25 years (12.2 \pm 9.4$)$.

The underlying theme and six major themes describing strategies to enhance the information, motivation, and skills affecting changes in diabetes self-management behavior of older adults are shown in Table 2. The underlying theme focused on the tone or implied feeling of the participants and was obtained by reviewing data within the context of the entire dataset for each participant. The major themes, as a check for specific instances of the categories, described the visible, surface, or obvious strategies of the participants' diabetes self-management. ${ }^{21}$

\section{Underlying theme}

The theme "one's own willingness and ability" emerged as a fundamental belief regarding diabetes self-management among older participants, which affected their experiences of managing diabetes. Both older adults and diabetes educators often emphasized the importance of "willingness and ability" in order to overcome the frustration from the fluctuation in blood glucose level and maintain diabetes self-management behaviors: 
Table 2 Underlying and major themes reported by study participants

\begin{tabular}{lll}
\hline Underlying theme & One's own willingness and ability & Motivation \\
\cline { 3 - 3 } Categories & Information & Ensuring a positive attitude \\
Major themes & Repeatedly offering detailed knowledge & regarding self-management \\
& regarding self-management & Encouragement or feedback \\
& Providing information about current health status & with numerical standards \\
& Identifying experiential knowledge of blood & \\
& glucose control & \\
\hline
\end{tabular}

Diabetes control is all in the mind, and having selfconfidence is such an important part of controlling my blood glucose level. [Group P2, older participant 1]

The patient's attitude is the important thing, thus it's very hard motivating a patient to perform self-management. Also, a patient who has confidence in controlling blood glucose takes care of his/her diabetes well. [Group E1, diabetes educator 2]

In these examples, both older adults and diabetes educators characterized their own willingness and ability as attitude and self-confidence toward diabetes self-management. These beliefs regarding the facilitation of diabetes self-management were seen as a result of implementing the specific strategies that enhanced the information, motivation, and skills.

\section{Major themes}

\section{Repeatedly offering detailed knowledge} regarding self-management

This theme described the participants' perspectives about the content and characteristics of DSME. Considering older adults' memory problems, DSME should be provided repeatedly and should include detailed information on performing diabetes self-management behaviors, such as diet, exercise, taking medication, and problem solving. Both older adults and diabetes educators often stated that the information was highly effective when provided by health professionals, such as physicians, nurses, dieticians, and exercise specialists:

If an exercise specialist tells me what kind of exercise or how much exercise would decrease my blood glucose level more, I would attend an exercise program more. Although I was once taught exercise by a specialist, I couldn't remember because of my poor memory. It is necessary that details of self-management were engraved on my memory through learning by repetition. [Group P1, older adult 7]

Fragmentary knowledge such as the kinds of diabetes complications is not effective, DSME should include details of self-management behaviors, for example what kind of foods, and how much they eat [...]. Specifically, the team approach composed of experts in each healthcare field could lead to more effective diabetes self-management because older adults tend to be more receptive to advice from an authoritative person. [Group E1, diabetes educator 1]

Both older adults and diabetes educators emphasized the detailed knowledge about diabetes self-management as an essential prerequisite for successful diabetes selfmanagement, as well as the need to repeat DSME, including details regarding diet and exercise practices explained by health professionals.

\section{Providing information about current health status}

Among the participants, this theme mostly appeared in the discussion by older diabetic adults. This theme also represented older diabetic adults' needs for tailored information considering their current health status, such as serum hemoglobin $\mathrm{A}_{1 \mathrm{c}}\left(\mathrm{HbA}_{1 \mathrm{c}}\right)$, cholesterol level, and comorbidity:

I didn't know until I got the explanation from a doctor of why medications are needed for my kidney and heart. So, I tried to be a lot more careful. [Group P1, older adult 2]

I felt good when my $\mathrm{HbA}_{1 \mathrm{c}}$ was low, but I felt bad when my $\mathrm{HbA}_{1 \mathrm{c}}$ was high. Then, I took time for self-reflection that I had a lot of sweets and alcohol these past couple of months and determined to drink less alcohol and eat healthy foods for my diabetes. [Group P1, older adult 7]

One participant in the educator group emphasized that information focusing on patients' self-monitoring of blood glucose was more important than providing other information by health professionals.

\section{Identifying experiential knowledge of blood glucose control}

Most participants perceived personal experience with blood glucose control as another source of knowledge for successful diabetes self-management. This experiential learning integrated a firm belief in their own way of managing diabetes, even if it was incorrect knowledge: 
I found my ways to control blood glucose level through frequent experience. I ate as much as I would like to, and got a lot of exercise to decrease my blood glucose level [...]. Then, I thought, let's eat heartily and more exercise. Also, red ginseng was the best I've ever had, because it was very effective in decreasing my blood glucose level. [Group P1, older adult 1]

It's very difficult for old adults to change their way of thinking about self-management behaviors because they stood firm in their belief through experience [...]. It can be a problem that no one corrects their misunderstanding, which has been formed by inaccurate information. [Group E1, diabetes educator 5]

There was a distinct difference between older adults and diabetes educators regarding "experiential knowledge." The majority of the older adults perceived experiential knowledge as a positive concept, while the diabetes educators thought more negatively about the older adults' experiential knowledge.

\section{Ensuring a positive attitude regarding self-management}

Older adults described a positive attitude regarding diabetes self-management as an effective strategy. This attitude often encouraged these patients to take an active part in diabetes care, such as participating in DSME and maintaining lifestyle modifications. The majority of the older adults perceived that diabetes self-management was necessary to live longer and remain independent in life:

I try to get diabetes education, and keep a good diet and exercise since I found that I would live healthily without complications by self-management. [Group P2, older adult 5]

I want to live long and healthy without help from others, and I do not wish to be a burden to my children. So, I walk an hour every day and try to eat less. [Group P1, older adult 3]

This attitude, which motivated older adults' selfmanagement, was primarily emphasized by the older adults, while the diabetes educators focused on the more technical aspects of teaching self-management, such as multidisciplinary team education or individualized goal setting.

\section{Encouragement or feedback from significant others}

Both older adults and diabetes educators often described support from a spouse, children, or health professionals, and many diabetes educators mentioned that health professionals' feedback regarding current self-management behaviors was especially helpful when patients were faced with a difficult problem. The older adults appreciated such support in exercise, diet, and taking medication:

Sometimes it's hard to wake up, and then my wife pushed me into taking medication, eating, and walking. So, again, I would go for a walk about one to two hours a day. [Group P1, older adult 4]

It's very important that health professionals offer words of encouragement or praise patients for the successful control of their glucose level. [Group E1, diabetes educator 1]

Despite efforts to manage diabetes in their own way, patients were faced with challenges such as frustration with blood glucose levels. In that case, pointing out the problem and offering a solution or alternative to current self-management behaviors would help patients change or persist with their method. [Group E1, diabetes educator 4]

Both older adults and diabetes educators emphasized the importance of support from family or health professionals regarding successful diabetes self-management. Older adults, in particular, needed this type of support when they were exhausted because of their frustration with their blood glucose level.

\section{Hands-on skills training with numerical standards}

Both older adults and diabetes educators demonstrated the need for hands-on skills training in exercise and diet regarding blood glucose control. Older adults in particular emphasized a method of self-management that was more tailored to individuals, using numerical standards for diet or exercise:

I knew for sure how to change my blood glucose levels depending on the amount of diet and exercise, because the diabetes education program provided opportunities for me to get hands-on experience through monitoring blood glucose levels before and after eating and exercise. [Group P2, older adult 5]

Before then, I used to eat a fist-sized portion of pork. But I'm trying to eat half a fist-sized portion of pork after learning that a fist-sized portion is too much for my blood glucose. [Group P1, older adult 7]

The experiential learning method is more effective, for instance, having a breakfast and exercise meeting where patients can experience diet-related or exercise-related changes in their blood glucose level. [Group E1, diabetes educator 2] 


\section{Discussion}

This qualitative study identified strategies for enhancing information, motivation, and skills necessary to improve diabetes self-management behaviors among communitydwelling older adults. The theme reinforcing "one's own willingness and ability" was delineated as a core strategy to improve self-management behaviors. This strategy means empowerment in disease management, taking responsibility for one's own health, and is an important belief to facilitate diabetes self-management behaviors among Korean older adults who have been influenced by Confucian culture that emphasize individuals' responsibility for their own behaviors and consequences. ${ }^{5}$ Therefore, empowerment support, particularly to encourage healthy diabetes-related behaviors and to address psychosocial concerns, ${ }^{10}$ can be more effective for Korean older adults.

Information, motivation, and skills were conceptualized as the process variables or core determinants for self-management behavior changes. ${ }^{11,22}$ In addition, optimal self-management requires the ability or support to use educational, psychosocial, and behavioral strategies to improve quality of life. ${ }^{23}$ In this study, the educational strategies for enhancing information necessary for diabetes self-management entailed repeatedly offering detailed knowledge about self-management, current health status, and identifying heuristics related to blood glucose control. These strategies will particularly help overcome aging-related problems, such as memory decline, poor understanding of diabetes self-management, and cognitive impairment. Furthermore, cognitive impairment is common in older adults with diabetes; ${ }^{24}$ nurses or diabetes educators working with older adults with diabetes need to consider older adults' cognitive functions, as this also influences poor health outcomes. The psychosocial strategies for enhancing motivation involved a positive attitude toward self-management behaviors or life in general and social support from family or health professionals. For older adults with diabetes, these can be important strategies in terms of psychosocial adjustment to diabetes, because social support has been identified as the most consistent factor related to it ${ }^{25}$ and has been associated with better glycemic control and selfmanagement. ${ }^{26}$ Moreover, social support is known to be a strong predictor of diabetes self-management behavior changes. ${ }^{27,28}$ However, there is now a growing trend for older adults to live alone or live solely with a spouse because of the low birthrate and rapidly aging population in Korea. In our study, many participants lived alone or lived solely with a spouse. It may be harder in the future for older adults who live alone or with an old or sick spouse to receive support from their families. A similar problem was reported in China regarding the longstanding "one-child" policy. ${ }^{18}$ Therefore, the strategy regarding social support within a community, such as peer group activities and senior center programs by health professionals in Korea, should focus on older adults who have no family. Peer support among older adults with diabetes or health professional support in community-based senior centers has reportedly improved self-management and health outcomes. ${ }^{29-31}$ Further, the behavioral strategies for enhancing skills should promote self-efficacy or confidence through hands-on skills training for blood glucose control. These strategies elicited from our study's focus group interview provided evidence of the need to develop a more tailored diabetes self-management program for older adults.

Other significant findings of this study were the similarities and differences of perceived strategies for diabetes selfmanagement between older adults with diabetes and diabetes educators. The similarities were that they emphasized the importance of repetitive DSME, including details for practice of diet and exercise by health professionals as an essential prerequisite for successful diabetes self-management. Moreover, support from family or health professionals for enhancing motivation was described by both groups. Considering that DSME is a core part of diabetes care and should be ongoing to promote the patients' knowledge, motivation, and skills necessary for diabetes self-management, ${ }^{32}$ this result is an inevitable consequence. Particularly, repetitive DSME is important considering that the effect of DSME was maintained only during a short period, lasting no more than 6 months. ${ }^{8}$ Older adults needed detailed and structured information and hands-on skills training in diet and exercise in particular, which is consistent with previous studies. ${ }^{15}$ Support from family or health professionals were factors motivating diabetes self-management behavior changes. Although this finding is similar to previous studies, ${ }^{5,15,16}$ the role of family and health professionals for managing older adults' diabetes was described in detail by the participants in this study. While the role of family is usually to provide verbal encouragement or reminders, the role of health professionals is to provide feedback on current problematic self-management behaviors and opportunities for hands-on skills training, including diet, exercise, and self-monitoring of blood glucose.

Apparent differences in strategies for diabetes selfmanagement between older adults and diabetes educators were found in three themes. While diabetic older adults described the importance of detailed explanations about their current 
health status from health professionals, diabetes educators emphasized patients' self-monitoring of blood glucose at home. In addition, the older adults who participated in this study reported the financial difficulty of buying the blood glucose meter and strips to measure their blood glucose level. This may be due to the medical insurance system in Korea, which does not cover glucose meters and strips, as well as the high relative poverty rate of older Korean adults. Therefore, nurses or diabetes educators working with older adults with diabetes must be sensitive to older adults' financial situations, as this also influences buying healthy foods or medication. Furthermore, diabetes educators should be mediators connecting diabetic older adults and community resources. ${ }^{10,33}$

There was also a difference of opinion regarding the experiential knowledge of blood glucose control. The majority of the older adults perceived experiential knowledge as a positive strategy, while diabetes educators thought more negatively about older adults' experiential knowledge. Although patients' incorrect knowledge was reported as a barrier to diabetes self-management by health professionals, ${ }^{34}$ endorsement of experiential knowledge of individuals has appeared to be strongly related to health behaviors. ${ }^{13}$ Therefore, further research investigating the types and effects of experiential knowledge, including folk remedies for managing diabetes in older adults, would be valuable. For example, red ginseng is the widely used herb for diabetes, although a systematic review concluded that the evidence for the effectiveness of red ginseng in controlling blood glucose is not valid. ${ }^{35}$

The third difference revealed in this study was that individual attitudes or beliefs for living with diabetes were primarily emphasized by the older adults, while diabetes educators focused on the more technical aspects of teaching self-management, such as a multidisciplinary team approach or accessibility of DSME. In previous studies, individual attitudes or beliefs, such as knowing the benefits of selfmanagement as the motivating factor, and goals for longevity and maintaining independence were found to be facilitators of diabetes self-management in older adults. ${ }^{5,16}$ Therefore, it is necessary that diabetes educators consider the content of DSME, reflecting the value of diabetic older adults, as well as the technical aspects of DSME.

The limitation of this study was that participants may not be representative of all community-dwelling older adults because they were sampled from only one geographic area in a large metropolitan area. Thus, the transferability of these findings to diabetes older adults in other regions might be limited. Nonetheless, the focus group interview approach of our study provided knowledge to develop a more culturally sensitive diabetes self-management program for older adults; indeed, programs that are based on elicitation research to empirically identify group-specific needs are more likely to be successful by ensuring the ecological validity. ${ }^{11}$

\section{Conclusion}

Strategies for promoting three important determinants information, motivation, and skills-ofdiabetes self-management behavior changes were identified in this study. These findings will contribute to developing a tailored and culturally sensitive diabetes self-management program with strategies for diabetes self-management for community-dwelling older adults.

\section{Acknowledgment}

This research was supported by the Basic Science Research Program through the National Research Foundation of Korea (NRF) funded by the Ministry of Education, Science and Technology (Grant No 2010-0022761).

\section{Disclosure}

The authors report no conflicts of interest in this work.

\section{References}

1. Brown AF, Mangione CM, Saliba D, Sarkisian CA. Guidelines for improving the care of the older person with diabetes mellitus. $J \mathrm{Am}$ Geriatr Soc. 2003;51(5 Suppl 1):S265-S280.

2. Wild S, Roglic G, Green A, Sicree R, King H. Global prevalence of diabetes: estimates for the year 2000 and projections for 2030. Diabetes Care. 2004;27(5):1047-1053.

3. Korea Centers for Disease Control and Prevention. Korea Health Statistics 2009: Korea National Health and Nutrition Examination Survey (KNHANESIV-3). Seoul: Korea Centers for Disease Control and Prevention; 2010.

4. Snoek FJ, Skinner TC, Steed L. Diabetes. In: Newman S, Steed L, Mulligan K, editors. Chronic Physical Illness: Self-Management and Behavioural Interventions. New York: McGraw-Hill; 2009: 169-188.

5. Song M, Lee M, Shim B. Barriers to and facilitators of self-management adherence in Korean older adults with type 2 diabetes. Int J Older People Nurs. 2010;5(3):211-218.

6. UK Prospective Diabetes Study Group. Intensive blood-glucose control with sulphonylureas or insulin compared with conventional treatment and risk of complications in patients with type 2 diabetes (UKPDS 33). Lancet. 1998;352(9131):837-853.

7. Hausmann LR, Ren D, Sevick MA. Racial differences in diabetesrelated psychosocial factors and glycemic control in patients with type 2 diabetes. Patient Prefer Adherence. 2010;4:291-299.

8. Norris SL, Lau J, Smith SJ, Schmid CH, Engelgau MM. Self-management education for adults with type 2 diabetes: a meta-analysis of the effect on glycemic control. Diabetes Care. 2002;25(7):1159-1171.

9. Coulter A, Ellins J. Changing attitudes to the role of patients in health care. In: Newman S, Steed L, Mulligan K, editors. Chronic Physical Illness: Self-Management and Behavioural Interventions. New York: McGraw-Hill; 2009:29-44.

10. Haas L, Maryniuk M, Beck J, et al. National standards for diabetes selfmanagement education and support. Diabetes Care. 2013;36(Suppl 1): S100-S108. 
11. Fisher JD, Fisher WA. Changing AIDS-risk behavior. Psychol Bull. 1992;111(3):455-474.

12. Fisher JD, Fisher WA, Amico KR, Harman JJ. An informationmotivation-behavioral skills model of adherence to antiretroviral therapy. Health Psychol. 2006;25(4):462-473.

13. Fisher JD, Fisher WA, Shuper PA. The information-motivationbehavioral skills model of HIV preventive behavior. In: DiClemente RJ, Crosby RA, Kegler MC, editors. Emerging Theories in Health Promotion Practice and Research. 2nd ed. San Francisco: Jossey-Bass; 2009:22-63.

14. Deakin T, McShane CE, Cade JE, Williams RD. Group based training for self-management strategies in people with type 2 diabetes mellitus. Cochrane Database Syst Rev. 2005;(2):CD003417.

15. Brewer-Lowry AN, Arcury TA, Bell RA, Quandt SA. Differentiating approaches to diabetes self-management of multi-ethnic rural older adults at the extremes of glycemic control. Gerontologist. 2010;50(5): 657-667.

16. Morrow AS, Haidet P, Skinner J, Naik AD. Integrating diabetes self-management with the health goals of older adults: a qualitative exploration. Patient Educ Couns. 2008;72(3):418-423.

17. McGuire AM, Anderson DJ. Lifestyle risk factor modification in midlife women with type 2 diabetes: theoretical modelling of perceived barriers. Aust J Adv Nurs. 2012;30(1):49-57.

18. Shen H, Edwards H, Courtney M, McDowell J, Wei J. Barriers and facilitators to diabetes self-management: perspectives of older community dwellers and health professionals in China. Int $J$ Nurs Pract. 2013;19:627-635.

19. Toobert DJ, Hampson SE, Glasgow RE. The summary of diabetes self-care activities measure: results from 7 studies and a revised scale. Diabetes Care. 2000;23(7):943-950.

20. Onwuegbuzie AJ, Dickinson WB, Leech NL, Zoran AG. A qualitative framework for collecting and analyzing data in focus group research. Int J Qual Methods. 2009;8(3):1-21.

21. Downe-Wamboldt B. Content analysis: method, applications, and issues. Health Care Women Int. 1992;13:313-321.

22. Ryan P, Sawin KJ. The individual and family self-management theory: background and perspectives on context, process, and outcomes. Nurs Outlook. 2009;57(4):217-225. e6.
23. Barlow J, Wright C, Sheasby J, Turner A, Hainsworth J. Selfmanagement approaches for people with chronic conditions: a review. Patient Educ Couns. 2002;48(2):177-187.

24. Nguyen HT, Kirk JK, Arcury TA, et al. Cognitive function is a risk for health literacy in older adults with diabetes. Diabetes Res Clin Pract. 2013;101(2):141-147.

25. Whittemore R, Melkus GDE, Grey M. Metabolic control, selfmanagement and psychosocial adjustment in women with type 2 diabetes. J Clin Nurs. 2005;14(2):195-203.

26. Akca AT, Cinar S. Comparison of psychosocial adjustment in people with diabetes with and without diabetic foot ulceration. Aust $J A d v$ Nurs. 2008;25(4):87-96.

27. Hunt CW, Wilder B, Steele MM, Grant JS, Pryor ER, Moneyham L. Relationships among self-efficacy, social support, social problem solving, and self-management in a rural sample living with type 2 diabetes mellitus. Res Theory Nurs Pract. 2012;26(2):126-141.

28. King DK, Glasgow RE, Toobert DJ, et al. Self-efficacy, problem solving, and social-environmental support are associated with diabetes self-management behaviors. Diabetes Care. 2010;33(4):751-753.

29. Smith SM, Paul G, Kelly A, Whitford DL, O'Shea E, O'Dowd T. Peer support for patients with type 2 diabetes: cluster randomised controlled trial. BMJ. 2011;342:d715.

30. Haltiwanger EP, Brutus H. A culturally sensitive diabetes peer support for older Mexican-Americans. Occup Ther Int. 2012;19(2):67-75.

31. Song M, Park Y-H, Song W, et al. Combined exercise training and self-management education for community-dwelling older adults with diabetes in Korea. J Gerontol Nurs. 2012;38(10):38-48.

32. Funnell MM, Brown TL, Childs BP, et al. National standards for diabetes self-management education. Diabetes Care. 2010;33 Suppl 1: S89-S96.

33. Nagelkerk J, Reick K, Meengs L. Perceived barriers and effective strategies to diabetes self-management. J Adv Nurs. 2006;54(2):151-158.

34. Lee YK, Ng CJ, Lee PY, et al. What are the barriers faced by patients using insulin? A qualitative study of Malaysian health care professionals' views. Patient Prefer Adherence. 2013;7:103-109.

35. Kim S, Shin BC, Lee MS, Lee H, Ernst E. Red ginseng for type 2 diabetes mellitus: a systematic review of randomized controlled trials. Chin J Integr Med. 2011;17(12):937-944.
Patient Preference and Adherence

\section{Publish your work in this journal}

Patient Preference and Adherence is an international, peer-reviewed, open access journal focusing on the growing importance of patient preference and adherence throughout the therapeutic continuum. Patient satisfaction, acceptability, quality of life, compliance, persistence and their role in developing new therapeutic modalities and compounds to

\section{Dovepress}

optimize clinical outcomes for existing disease states are major areas of interest. This journal has been accepted for indexing on PubMed Central. The manuscript management system is completely online and includes a very quick and fair peer-review system. Visit http://www.dovepress.com/ testimonials.php to read real quotes from published authors. 